\title{
Efeitos da Exposição a Dispositivos Digitais no Desenvolvimento da Linguagem em Idade Pré-Escolar
}

\section{Effects of Exposure to Digital Devices on Language Development of Preschoolers}

Rita Neto ${ }^{1}$, Daniel Dias Gonçalves²

Autor Correspondente/Corresponding Author:

Rita Neto [mimed0602233@med.up.pt]

\section{RESUMO}

Nas últimas décadas, os dispositivos digitais tornaram-se parte integrante do nosso quotidiano. Atualmente, crianças com menos de 1 ano já são expostas a conteúdos media. $\bigcirc$ impacto que estes novos hábitos têm no desenvolvimento da linguagem em crianças de idade pré-escolar não é claro. Neste artigo de revisão estudámos os trabalhos mais relevantes sobre este tema publicados após o ano 2000. Dos 10 artigos selecionados, sete analisaram o tempo de exposição e cinco o tipo de conteúdos media. Cinco artigos encontraram uma associação negativa entre o tempo de exposição a dispositivos digitais e o desenvolvimento da linguagem, enquanto apenas dois encontraram benefícios. Dos cinco artigos que analisaram o conteúdo media apenas um encontrou uma associação positiva entre a visualização de conteúdo educativo para crianças e a linguagem. Assim, os autores recomendam que, em idade pré-escolar, os dispositivos digitais sejam usados de forma parcimoniosa e acompanhada pelos pais.

PALAVRAS-CHAVE: Computadores; Desenvolvimento Infantil; Desenvolvimento da Linguagem; Mass Media; Pré-Escolar; Smartphone; Televisão 


\section{ABSTRACT}

In the last decades, digital devices have become a central part in our lives. Nowadays, infants are already exposed to a handful of digital media. It is unclear what's the impact of these new habits on preschoolers' language development. Here, we have reviewed the most relevant papers published on this subject since the year 2000. From the 10 selected papers, seven analyzed the duration of exposure and five the type of media content. Five papers found a negative association between the duration of exposure to digital devices and language development, while only two papers found benefits. From the five papers analyzing media content only one showed a positive association between visualization of educational programs and a healthy language development. The authors recommend limited and parent-accompanied usage of digital devices by preschoolers.

KEYWORDS: Child Development; Child, Preschool; Computers; Language Development; Mass Media; Smartphone; Television

\section{INTRODUÇÃO}

Nas últimas décadas, a utilização de tablets e smartphones massificou-se e alterou profundamente os hábitos de vida da população. Desde 1983, altura em que chegou ao mercado o primeiro telemóvel, ${ }^{1}$ os dispositivos móveis tornaram-se cada vez mais acessíveis e centrais no nosso dia-a-dia. Se em 2011 apenas 8\% das famílias americanas com crianças até aos 8 anos tinham tablet(s) e 41\% smartphone(s), em 2017 a percentagem atingia os $78 \%$ e $95 \%$, respetivamente. ${ }^{2}$ Atualmente um adulto utiliza o smartphone, em média, 2 horas e 39 minutos por dia. ${ }^{3}$ A ubiquidade destes dispositivos, concomitantemente com os formatos media mais tradicionais (televisão e computador), traduz-se também numa utilização cada vez mais precoce dos mesmos. Vivemos num mundo em que "nativos digitais" são criados e educados por "imigrantes digitais". ${ }^{2}$ Desde 1970, a idade com que uma criança começa a interagir regularmente com os media passou dos 4 anos para os 4 meses. ${ }^{5}$ As consequências destes novos hábitos ainda não são perfeitamente conhecidas, mas já são uma preocupação entre pais, professores e profissionais de saúde, que querem saber se e como regular o uso das novas tecnologias junto das suas crianças.

Os dispositivos digitais (DD) comportam um potencial educativo importante, que pode assumir um papel influente no desenvolvimento da criança. Contudo, a sua utilização não parece ser isenta de riscos. Em crianças em idade escolar, a exposição a DD foi associada a pior qualidade do sono, ${ }^{6,7}$ da acuidade visual,, 7.8 do bem-estar emocional e das funções executivas ${ }^{7,9,10}$ e a obesidade., ${ }^{7,11}$ Uma vez que atualmente a maioria das crianças contacta com um DD durante o primeiro ano de vida, ${ }^{12}$ é hoje relevante questionar qual o impacto da sua utilização no desenvolvimento cognitivo e emocional de crianças ainda mais jovens.

É durante os 3 primeiros anos de vida que o cérebro se desenvolve mais rapidamente, ${ }^{13}$ e é este também o pe- ríodo de maior recetividade e vulnerabilidade aos estímulos do meio envolvente. ${ }^{14} \mathrm{O}$ desenvolvimento da linguagem, de competências motoras e o estabelecimento das primeiras relações sociais e afetivas ocorrem durante o período pré-escolar. ${ }^{5,15}$ Especificamente, a aquisição da linguagem é um processo complexo e indispensável ao desenvolvimento harmonioso de uma criança, e que depende essencialmente da exposição precoce a ambientes verbalmente estimulantes. ${ }^{16} \mathrm{~A}$ atual alteração dos hábitos de vida, associados à utilização massiva de DD, influencia diretamente a forma como comunicamos e pode afetar o desenvolvimento da linguagem de crianças pré-verbais.

Perante os potenciais riscos associados, a Associação Americana de Pediatria atualizou em 2016 a sua posição relativamente ao tempo máximo que os DD devem ocupar no dia de uma criança. Sugerem que: até aos 24 meses deve ser evitado qualquer DD (com exceção de aplicações de videoconferência); pais de crianças entre os 18-24 meses, que optem pela utilização de DD, devem escolher programas de alta qualidade, adaptados à idade e são aconselhados a assistirem aos programas com as crianças, para que elas possam compreender o que estão a assistir; crianças entre os 2 e os 5 anos não devem utilizar DD mais de 1 hora por dia, idealmente também acompanhados pelos pais ou cuidadores. ${ }^{17} \mathrm{No}$ entanto, no estudo de Kabali et al de 2015, verifica-se que a exposição pré-escolar aos media ultrapassa largamente as recomendações: praticamente metade (43,5\%) das crianças com menos de 1 ano utilizam dispositivos móveis diariamente para jogar, ver vídeos ou utilizar aplicações; aos 2 anos, esta percentagem aumenta para $76,6 \%$ das crianças e atinge os $81,6 \%$ aos 4 anos. ${ }^{12}$ Os resultados do Common Sense Census de 2017, 2 o último de uma série de três inquéritos com dados de uma amostra americana representativa a nível nacional, mostram resultados semelhantes. Os padrões de consumo modificaram-se bastante ao longo das 3 fases do estudo, particularmente nos dispositivos utilizados. Apesar da 
quase manutenção do tempo de utilização de DD (utilização média diária de 2 horas e 19 minutos em 2017 versus 2 horas e 16 minutos em 2011), observou-se um decréscimo no tempo dedicado a ver televisão e DVDs e um aumento no tempo de utilização diário de dispositivos móveis (5 minutos em 2011 versus 48 minutos em 2017). Ainda assim, a visualização de vídeos (programas de televisão, filmes, etc.), quer através de dispositivos móveis ou por meios mais convencionais, mantém-se como a atividade dominante, ocupando cerca de $72 \%$ de todo o tempo de ecrã. Não existem estudos detalhados sobre a evolução e consumo de DD na Europa.

Neste contexto, este artigo de revisão propõe-se a rever o que se sabe até à data sobre o impacto da exposição e utilização de DD no desenvolvimento da linguagem de lactentes e crianças em idade pré-escolar.

\section{MATERIAL E MÉTODOS}

As plataformas media que hoje em dia nos chegam via ecrãs incluem a televisão, os computadores, as consolas de jogos e os dispositivos móveis, essencialmente representados por smartphones e tablets. Neste estudo, todos os modelos de exposição foram incluídos e serão referidos como DD.

Os autores realizaram uma pesquisa bibliográfica em fevereiro de 2019 de artigos exclusivamente escritos em inglês ou português, desde 1 de janeiro de 2000 até à data, através da plataforma PubMed. Foram utilizados os seguintes termos MeSH: "infant", "child, preschool", "screen time", "television", "smartphone", "computers", "computers, handheld", "child development", "language development" e "language development disorders", tendo-se adotado a seguinte estratégia de pesquisa: (infant OR child, preschool) AND (screen time OR television OR smartphone OR computers, handheld OR computers) AND (language development OR language development disorders). Foi acrescentada à pesquisa inicial uma pesquisa manual de referências bibliográficas que pudesse identificar estudos relevantes que não teriam sido incluídos previamente.

Os critérios de inclusão utilizados foram: estudos originais; população constituída por crianças até aos 6 anos; exposição a DD quantificada em tempo de exposição total ou tempo de exposição a diferentes conteúdos digitais e avaliação do desenvolvimento da linguagem como outcome primário, objetivado através de escalas de avaliação de linguagem adaptadas à idade da criança. Os critérios de exclusão utilizados foram: população com mais de 6 anos e crianças com diagnóstico prévio de perturbações do neurodesenvolvimento.

\section{RESULTADOS}

Foram encontrados 334 artigos pela metodologia de pesquisa anteriormente descrita, 331 encontrados na plataforma PubMed e três por pesquisa manual. Destes, foram selecionados $10 \mathrm{com}$ base nos critérios de inclusão e exclusão descritos e mediante análise crítica e discussão entre os autores. A Fig. 1 representa o fluxograma de seleção dos estudos. Não foi aplicado nenhum método estatístico na análise dos artigos selecionados.

Dos 10 artigos selecionados, seis analisaram amostras de crianças americanas, ${ }^{18-23}$ três de diferentes populações asiáticas (Japão, ${ }^{24}$ Coreia do Sul ${ }^{25}$ e Tailândia ${ }^{26}$ ) e um de crianças australianas. ${ }^{27}$ Apesar dos diferentes desenhos de estudo e tamanhos amostrais, a exposição a DD foi quantificada de forma semelhante, mediante a aplicação de questionários de exposição típica ou diários acerca das 24 horas anteriores aos pais ou cuidadores das crianças. $\bigcirc$ desenvolvimento da linguagem foi determinado através de diferentes escalas de avaliação e inclui resultados sobre o desempenho a vários níveis linguísticos, como o reconhecimento de letras e palavras, o vocabulário compreendido e a capacidade de comunicação expressiva.

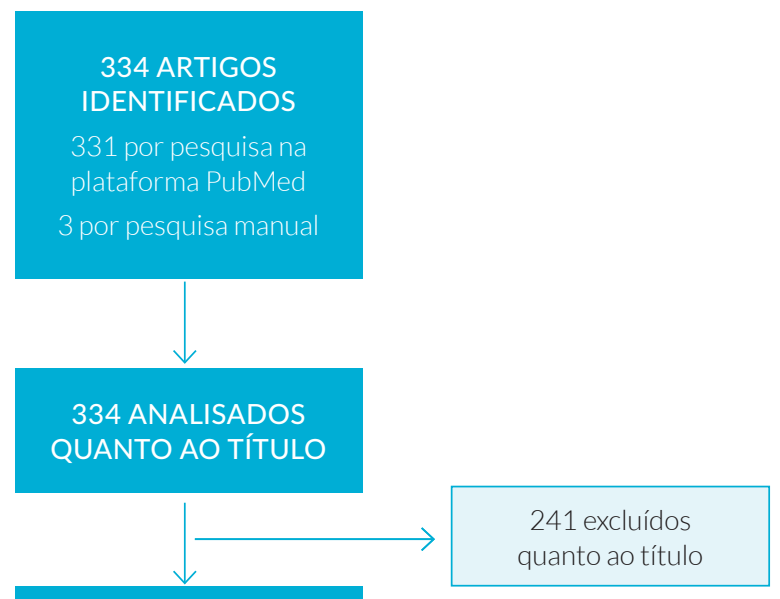

93 ANALISADOS QUANTO AO ABSTRACT

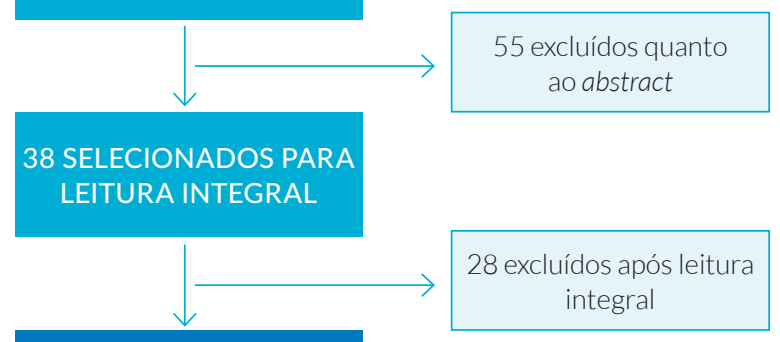

10 ARTIGOS INCLUÍDOS

FIGURA 1. Fluxograma de seleção dos estudos. 
TABELA 1. Descrição dos estudos incluídos com resultados sobre o tempo de exposição.

\begin{tabular}{|c|c|c|c|c|c|c|c|c|}
\hline Artigo & Ano & País & $\begin{array}{l}\text { Tipo de } \\
\text { Estudo }\end{array}$ & $\begin{array}{l}\text { Exposição (medida } \\
\text { de quantificação) }\end{array}$ & Idades & Amostra & Outcomes (Teste) & Principais resultados \\
\hline \multirow{2}{*}{$\begin{array}{l}\text { Zimmerman } \\
\text { et al. }\end{array}$} & \multirow[b]{2}{*}{2005} & \multirow{2}{*}{ EUA } & \multirow{2}{*}{$\begin{array}{l}\text { Longitudinal } \\
\text { retrospetivo }\end{array}$} & \multirow{2}{*}{$\begin{array}{c}\text { Televisão } \\
\text { (questionário de } \\
\text { exposição típica/dia) }\end{array}$} & \multirow{2}{*}{$\begin{array}{c}\text { Exposição: } \\
\text { < } 6 \text { anos } \\
\text { Outcome: } \\
6 \text { anos }\end{array}$} & 1795 & $\begin{array}{c}\text { Reconhecimento } \\
\text { de letras/palavras } \\
\text { (PIAT) })^{*}\end{array}$ & $\begin{array}{c}\text { Exposição < } 3 \text { anos: } \\
\text { associação negativa } \\
\text { com } 2 \text { outcomes; }\end{array}$ \\
\hline & & & & & & 1034 & $\begin{array}{l}\text { Compreensão } \\
\text { escrita (PIAT)* }\end{array}$ & $\begin{array}{c}\text { Exposição 3-5 anos: } \\
\text { associação positiva } \\
\text { com reconhecimento } \\
\text { de letras/palavras }\end{array}$ \\
\hline Linet al. & 2015 & Tailândia & Transversal & $\begin{array}{c}\text { Televisão, } \\
\text { smartphone, tablet, } \\
\text { computador } \\
\text { (entrevista com } \\
\text { cuidador) }\end{array}$ & $\begin{array}{l}15-35 \\
\text { meses }\end{array}$ & $\begin{array}{c}\text { 150: } \\
75 \text { (alta } \\
\text { exposição) } \\
+75 \text { (baixa } \\
\text { exposição) }\end{array}$ & $\begin{array}{l}\text { Linguagem } \\
(\text { BSID-II)** }\end{array}$ & $\begin{array}{c}\text { Exposição a DD } \\
\text { aumenta 3,3 o risco } \\
\text { de desenvolvimento } \\
\text { de perturbações da } \\
\text { linguagem } \\
\text { (IC 95\%: 1,5-7,3) }\end{array}$ \\
\hline \multirow{2}{*}{ Duchet al. } & \multirow{2}{*}{2013} & \multirow{2}{*}{$\begin{array}{c}\text { EUA } \\
\text { (amostra } \\
\text { hispânica) }\end{array}$} & Transversal & \multirow{2}{*}{$\begin{array}{l}\text { Televisão, telemóveis, } \\
\text { DVDs, computador } \\
\text { (diário 24h- } \\
\text { anteriores) }\end{array}$} & \multirow[t]{2}{*}{$<3$ anos } & 119 & \multirow{2}{*}{$\begin{array}{l}\text { Comunicação } \\
\text { (ASQ3)* }^{*}\end{array}$} & $\begin{array}{c}>2 \text { horas/dia de } \\
\text { DD foi associado a } \\
\text { piores resultados no } \\
\text { outcome }(\beta=-1,71, P \\
=0,03)\end{array}$ \\
\hline & & & $\begin{array}{l}\text { Longitudinal } \\
\text { (follow-up } \\
1 \text { ano) }\end{array}$ & & & 73 & & $\begin{array}{c}>2 \text { horas/dia foi } \\
\text { associado a piores } \\
\text { resultados no } \\
\text { outcome }(B=-1,49, P \\
=0,008)\end{array}$ \\
\hline $\begin{array}{l}\text { Tomopoulus } \\
\text { et al. }\end{array}$ & 2010 & EUA & $\begin{array}{l}\text { Longitudinal } \\
\text { prospetivo }\end{array}$ & $\begin{array}{c}\text { Televisão, vídeos, } \\
\text { DVDs, jogos (diário } \\
\text { 24h- anteriores) }\end{array}$ & $\begin{array}{l}6 \text { meses: } \\
\text { exposição: } \\
14 \text { meses: } \\
\text { outcome }\end{array}$ & 259 & $\begin{array}{c}\text { Compreensão } \\
\text { auditiva + } \\
\text { Comunicação } \\
\text { expressiva } \\
\left(\text { PLS-4) }{ }^{+}\right.\end{array}$ & $\begin{array}{c}\text { Associação negativa } \\
\text { entre duração } \\
\text { da exposição e } 2 \\
\text { outcomes (efeito } \\
\text { mais forte para } \\
\text { compreensão } \\
\text { auditiva) }\end{array}$ \\
\hline Moonet al. & 2018 & $\begin{array}{l}\text { Coreia do } \\
\text { Sul }\end{array}$ & Transversal & $\begin{array}{l}\text { Smartphone, tablet } \\
\text { (questionário de } \\
\text { exposição típica) }\end{array}$ & 3-5 anos & 117 & $\begin{array}{c}\text { (Preschool receptive } \\
\text { expressive language } \\
\text { scale) }\end{array}$ & $\begin{array}{c}\text { Associação negativa } \\
\text { entre exposição aos } \\
3 \text { anos e linguagem } \\
\text { expressiva }\end{array}$ \\
\hline $\begin{array}{l}\text { Schmidt } \\
\text { et al. }\end{array}$ & 2009 & EUA & $\begin{array}{l}\text { Longitudinal } \\
\text { prospetivo }\end{array}$ & $\begin{array}{l}\text { Televisão, vídeos } \\
\text { (questionário de } \\
\text { exposição típica/dia) }\end{array}$ & $\begin{array}{l}\text { Exposição: } \\
\text { 0-2 anos } \\
\text { Outcome: } \\
3 \text { anos }\end{array}$ & 872 & $\begin{array}{l}\text { Vocabulário } \\
\text { compreendido } \\
(\text { PPVT-III)** }\end{array}$ & $\begin{array}{c}\text { Exposição até aos } 2 \\
\text { anos não se associa } \\
\text { ao outcome aos } 3 \\
\text { anos }\end{array}$ \\
\hline Castles et al. & 2013 & Austrália & Transversal & $\begin{array}{c}\text { Televisão, } \\
\text { computador } \\
\text { (questionário de } \\
\text { exposição típica/dia) }\end{array}$ & 4 anos & 1539 & $\begin{array}{l}\text { - Conhecimento das } \\
\text { letras }^{\dagger} \\
\text { - Linguagem oral } \\
\text { (CELF) } \\
\text { - Articulação } \\
\text { (GFTA) } \\
\text { - Reconhecimento } \\
\text { fonológico (SPAT) }\end{array}$ & $\begin{array}{c}\text { Associação positiva } \\
\text { entre utilização } \\
\text { de computador e } \\
\text { conhecimento das } \\
\text { letras }\end{array}$ \\
\hline
\end{tabular}

ASQ3: Ages and Stages Questionnaire, Third Edition; BSID-II: Bayley Scale of Infant Development, Second Edition; CELF: Clinical Evaluation of Language Fundamentals - Preschool, Second Edition; GFTA: Goldman Fristoe Test of Articulation; PIAT: Peabody Individual Achievement Test; PLS-4: Preschool Language Scale, Fourth Edition; PPVT-III: Peabody Picture Vocabulary Test III; SPAT: Sutherland Phonological Awareness Test; *aplicado por pais/cuidadores primários; **aplicado por profissionais de saúde; †aplicação não referenciada no artigo

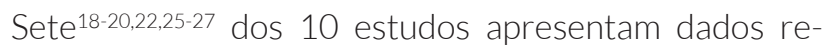
lativos ao impacto do tempo de exposição a DD no desempenho cognitivo (Tabela 1). Destes, quatro $18,20,25,26$ associam-no negativamente com os resultados, um $^{27}$ revela uma associação positiva entre a utilização de computador e o conhecimento de letras em crianças de 4 anos, um ${ }^{19}$ não estabelece associação entre a exposição e o outcome primário e um²2 apresenta uma associação negativa entre o tempo de visualização de televisão antes dos 3 anos com a capacidade de reconhecimento de letras/palavras e de compreensão escrita, ambas avaliadas aos 6 anos, e uma associação positiva entre ver televisão entre os 3 e os 5 anos e o reconhecimento de letras/palavras aos 6 anos, sem efeito sobre a compreensão escrita.

Metade 18,20,21,23,24 dos estudos selecionados apresenta resultados quanto ao impacto dos diferentes tipos de conteúdo a que as crianças são expostas nas suas capacidades cognitivas (Tabela 2). Os estudos apresentam diferentes categorias para o tipo de conteúdos digitais, como programação para crianças ou para público geral, programação educativa ou não educativa, etc. 
TABELA 2. Descrição dos estudos incluídos com resultados sobre os tipos de conteúdo.

\begin{tabular}{|c|c|c|c|c|c|c|c|c|c|}
\hline Artigo & Ano & País & $\begin{array}{l}\text { Tipo de } \\
\text { Estudo }\end{array}$ & $\begin{array}{c}\text { Exposição } \\
\text { (medida de } \\
\text { quantificação) }\end{array}$ & $\begin{array}{l}\text { Tipos de conteúdo } \\
\text { media }\end{array}$ & Idades & Amostra & $\begin{array}{l}\text { Outcomes } \\
\text { (Teste) }\end{array}$ & Principais resultados \\
\hline $\begin{array}{l}\text { Wright } \\
\text { etal. }\end{array}$ & 2001 & EUA & $\begin{array}{l}\text { Longitudinal } \\
\text { prospetivo } \\
\text { (follow-up } 3 \\
\text { ano) }\end{array}$ & $\begin{array}{l}\text { Televisão (diários } \\
\text { 24h- anteriores) }\end{array}$ & $\begin{array}{l}\text { Educativo para } \\
\text { crianças (1), } \\
\text { Não educativo } \\
\text { (animação) para } \\
\text { crianças (2), } \\
\text { Outros para cri- } \\
\text { anças (3), Público } \\
\text { geral (4) }\end{array}$ & $\begin{array}{c}\text { Coorte } \\
\text { 1: } \\
2 \text { anos } \\
\text { no início } \\
\text { do } \\
\text { estudo } \\
\\
\\
\text { Coorte } \\
\text { 2: } \\
4 \text { anos } \\
\text { no início } \\
\text { do } \\
\text { estudo }\end{array}$ & 92 & $\begin{array}{l}\text { Reconhec- } \\
\text { imento de } \\
\text { letras/palavras } \\
(\text { WJTA) } \\
+ \\
\text { Vocabulário } \\
\text { (PPVT-R)** }\end{array}$ & $\begin{array}{c}\text { (1) : entre } 2 \text {-3 anos } \\
\text { associou-se a melhores } \\
\text { outcomes aos } 3 \text { anos } \\
\text { nos } 2 \text { testes } \\
\text { (2): entre } 2 \text {-3 anos } \\
\text { associou-se a pior } \\
\text { outcome no WJTA } \\
\text { aos } 3 \text { anos } \\
\text { (4): entre } 2 \text {-3 anos } \\
\text { associou-se a pior out- } \\
\text { come no PPVT-R } \\
\text { aos } 3 \text { anos e entre os 4-5 } \\
\text { anos associou-se a pior } \\
\text { outcome no WJTA } \\
\text { aos } 5 \text { anos }\end{array}$ \\
\hline $\begin{array}{l}\text { Zimmer- } \\
\text { man } \\
\text { etal. }\end{array}$ & 2007 & EUA & Transversal & $\begin{array}{c}\text { Televisão, } \\
\text { DVDs, Vídeos } \\
\text { (questionário de } \\
\text { exposição típica) }\end{array}$ & $\begin{array}{c}\text { Educativo para lac- } \\
\text { tentes, Educativo } \\
\text { para crianças, Não } \\
\text { educativo para } \\
\text { crianças, Público } \\
\text { geral }\end{array}$ & $\begin{array}{c}\text { 8-24 } \\
\text { meses }\end{array}$ & 1008 & $\begin{array}{l}\text { 8-16 meses: } \\
\text { linguagem } \\
\text { compreendida } \\
+ \\
\text { 17-24 meses: } \\
\text { linguagem } \\
\text { expressiva } \\
\text { (MB-CDI)* }^{*}\end{array}$ & $\begin{array}{c}\text { Visualização de } \\
\text { programas para lactentes } \\
\text { em crianças dos 8-16 } \\
\text { meses associou-se } \\
\text { negativamente ao } \\
\text { outcome. } \\
\text { Não foram encontradas } \\
\text { associações significativas } \\
\text { para outros tipos } \\
\text { de conteúdo }\end{array}$ \\
\hline $\begin{array}{l}\text { Okuma } \\
\text { etal. }\end{array}$ & 2009 & Japão & Transversal & $\begin{array}{c}\text { Televisão, Vídeos } \\
\text { DVDs } \\
\text { (questionário de } \\
\text { exposição típica, } \\
\text { incluindo vídeos } \\
\text { favoritos) }\end{array}$ & $\begin{array}{c}\text { Educativos para } \\
\text { lactentes, Educati- } \\
\text { vos para crianças, } \\
\text { Programa infantil, } \\
\text { Cantar e dançar, } \\
\text { Animação simples, } \\
\text { Animação realista } \\
\text { (curta e longa), } \\
\text { Veículos, Animais, } \\
\text { Outros }\end{array}$ & $\begin{array}{c}18 \\
\text { meses }\end{array}$ & 378 & IMDS* $^{*}$ & $\begin{array}{c}\text { Grupo de crianças com } \\
\text { perturbação da } \\
\text { linguagem (pelo IMDS) } \\
\text { prefere Animação } \\
\text { realista (curta e longa) } \\
\text { e vídeos educativos para } \\
\text { lactentes }\end{array}$ \\
\hline $\begin{array}{l}\text { To- } \\
\text { mopou- } \\
\text { lus et al. }\end{array}$ & 2010 & EUA & $\begin{array}{l}\text { Longitudinal } \\
\text { prospetivo }\end{array}$ & $\begin{array}{l}\text { Televisão, vídeos, } \\
\text { DVDs, jogos } \\
\text { (diário 24h-an- } \\
\text { teriores) }\end{array}$ & $\begin{array}{c}\text { Programação para } \\
\text { crianças em idade } \\
\text { escolar/Público } \\
\text { geral, Educativo } \\
\text { para crianças/ } \\
\text { lactentes, Não } \\
\text { educativo para } \\
\text { crianças }\end{array}$ & $\begin{array}{l}6 \text { meses: } \\
\text { ex- } \\
\text { posição } \\
14 \\
\text { meses: } \\
\text { outcome }\end{array}$ & 259 & $\begin{array}{l}\text { Compreensão } \\
\text { auditiva }+ \\
\text { Comunicação } \\
\text { expressiva } \\
(\text { PLS-4)† }\end{array}$ & $\begin{array}{c}\text { Associação negativa } \\
\text { entre exposição a pro- } \\
\text { gramação para crianças } \\
\text { em idade escolar/público } \\
\text { geral com outcomes }\end{array}$ \\
\hline $\begin{array}{l}\text { Duchet } \\
\text { al. }\end{array}$ & 2013 & $E \cup A^{\S}$ & $\begin{array}{l}\text { Longitudinal } \\
\text { (follow-up } \\
1 \text { ano) }\end{array}$ & $\begin{array}{l}\text { Televisão, } \\
\text { telemóveis, } \\
\text { DVDs, } \\
\text { computador } \\
\text { (diário 24h } \\
\text { anteriores) }\end{array}$ & $\begin{array}{c}\text { Programação } \\
\text { para crianças, } \\
\text { Programação para } \\
\text { Público geral }\end{array}$ & $<3$ anos & 119 & $\begin{array}{l}\text { Comunicação } \\
\text { (ASQ3)* }^{*}\end{array}$ & $\begin{array}{c}\text { Associação negativa } \\
\text { entre exposição } \\
\text { a programação para } \\
\text { crianças com outcome; } \\
\text { Programação para } \\
\text { Público geral não foi } \\
\text { associada ao outcome }\end{array}$ \\
\hline
\end{tabular}

ASQ3: Ages and Stages Questionnaire, Third Edition; IMDS: Infant Mental Development Scale; MB-CDI: MacArthur-Bates Communicative Development Inventory; PLS-4: Preschool Language Scale, Fourth Edition; PPVT-R: Peabody Picture Vocabulary Test - Revised; WJTA: Woodcock-Johnson Tests of Achievement; *aplicado por pais/cuidadores primários; **aplicado por profissionais de saúde; ’aplicação não referenciada no artigo; ${ }^{\S}$ amostra hispânica

Quanto ao impacto da visualização de conteúdo educativo para crianças, um estudo ${ }^{21}$ encontrou uma associação positiva entre esta exposição entre os 2 e os 3 anos com os resultados linguísticos avaliados; dois estudos ${ }^{23,24}$ não encontram associações estatisticamente significativas e dois estudos ${ }^{18,20}$ não referem especificamente esta variável. Apenas dois estudos ${ }^{23,24}$ apresentam resultados quanto ao impacto da visualização de conteúdo educativo dirigido exclusivamente a lactentes e ambos apresentam associações negativas entre esta exposição com os resultados linguísticos. Relativamente a conteúdos não educativos para crianças, dois estudos ${ }^{21,24}$ apontam para uma associação negativa com o desenvolvimento linguístico, dois estudos ${ }^{20,23}$ não encontram associação entre as variáveis e um ${ }^{18}$ não refere especificamente esta variável. Dois estudos ${ }^{20,21}$ apontam para uma associação negativa entre a visualização de conteúdo concebido para o público geral com o desempenho linguístico; dois estudos $^{18,23}$ não encontram associações entre as variáveis e um estudo ${ }^{24}$ não menciona esta exposição. 


\section{DISCUSSÃO}

Neste artigo propusemo-nos a clarificar o efeito da utilização de DD no desenvolvimento da linguagem em idade pré-escolar. Por forma a facilitar o estudo dos seus efeitos, o "tempo de exposição" e o "conteúdo" foram tratados como variáveis distintas.

Globalmente, os estudos mostram que a exposição a dispositivos digitais se associa a um efeito negativo no desenvolvimento da linguagem.

A maioria dos estudos selecionados para inclusão nesta revisão mostram uma associação negativa entre o tempo de exposição a DD e o desenvolvimento da linguagem em crianças de idade pré-escolar. O consumo de DD associou-se a piores resultados no reconhecimento de letras/palavras, compreensão escrita e auditiva e linguagem expressiva. ${ }^{18,20,22,25,26}$ Dos 7 estudos analisados,

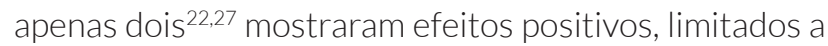
uma subfaixa etária e apenas para o reconhecimento de letras/palavras. Todos os efeitos negativos no desenvolvimento da linguagem foram observados quando a exposição aos DD ocorreu até aos 3 anos, ${ }^{18,20,22,25,26}$ e todos os efeitos positivos foram observados em crianças com mais de 3 anos. ${ }^{22,27}$ Alguns autores sugeriram que este efeito poderá ser devido ao tipo de conteúdo mais visualizado nas diferentes faixas etárias. No entanto, também é plausível que as diferenças advenham das fases distintas de aquisição da linguagem, da maior suscetibilidade aos estímulos pelas crianças mais jovens e da sua incapacidade de escolher os conteúdos a que são expostas. Um estudo dos 7 não encontrou nenhuma associação entre consumo de DD e linguagem. ${ }^{19}$ Apesar dos seus resultados iniciais (apenas ajustados para a idade e sexo da criança) mostrarem uma associação negativa entre as variáveis, esta desaparece quando as análises são ajustadas para características maternas relevantes, nomeadamente idade, rendimento, educação, estado civil e paridade, sugerindo que estes determinantes justifiquem as diferenças previamente encontradas.

A medição do tempo de exposição a DD apresenta alguns desafios, consequência da ubiquidade destes dispositivos e da sua utilização autónoma por crianças cada vez mais jovens, ${ }^{12}$ o que dificulta a quantificação do efeito e a sua comparação entre estudos. Adicionalmente, diferentes métodos foram usados quer para medir a exposição a DD quer para quantificar o desenvolvimento da linguagem. Globalmente, os estudos que comparam crianças saudáveis com diferentes níveis de exposição a DD mostraram um efeito modesto, mas estatisticamente significativo nos testes da linguagem, que se manteve mesmo após análise para possíveis variáveis de confun- dimento. Dos estudos incluídos, destacam-se alguns que encontraram um efeito de dose-resposta, sugestivo de causalidade.20,22,25 Estudos em crianças com perturbação da linguagem ${ }^{28}$ corroboram esta observação, já que estas começam a ver televisão mais cedo (7 vs 12 meses) e veem mais televisão (3 vs 2 horas/dia) que crianças com desenvolvimento normativo para a idade.

Se por um lado a ausência de um método padrão para quantificar o desenvolvimento da linguagem prejudica a comparação entre estudos, por outro a existência de estudos que, usando diferentes métodos, obtiveram achados na mesma direção aumenta a confiança no efeito. Adicionalmente, resultados semelhantes foram obtidos em diferentes populações, nomeadamente a americana ${ }^{18,20,22}$ e a asiática. ${ }^{25,26}$

A maioria dos programas educativos não parece promover o desenvolvimento da linguagem.

Muito do conteúdo digital dirigido a lactentes e crianças em idade pré-escolar reivindica enorme potencial no desenvolvimento das capacidades cognitivas e linguísticas junto do seu público alvo ${ }^{29}$ e a grande maioria dos pais acredita que este tipo de conteúdos é benéfico. ${ }^{18,30}$ No entanto, estas reivindicações não parecem ter evidência que as suporte. Neste trabalho incluímos cinco estudos que detalharam o tipo de conteúdo a que as crianças foram expostas. Destes, apenas um encontrou benefício na visualização de programas educativos entre os $2 \mathrm{e}$ os 3 anos e o vocabulário compreendido aos 3 anos. ${ }^{21}$ Os outros quatro ou não encontraram associação ou encontraram um efeito negativo da exposição a programas educativos. ${ }^{18,20,23,24}$ Não encontrámos dados consistentes que apontem para uma diferença real entre programas educativos dirigidos a lactentes ou a crianças em idade pré-escolar. Contudo, podem existir diferenças que sejam dependentes das características específicas de cada programa e não da sua categoria. Neste sentido, Linebarger e Walker ${ }^{31}$ mostraram que programas como "Dora, a Exploradora" e "As pistas da Blue" se associam a níveis superiores de vocabulário e linguagem expressiva, enquanto a "Rua Sésamo" e os "Teletubbies" foram negativamente associados aos mesmos parâmetros. ${ }^{31}$ Relativamente à visualização de conteúdo não dirigido a crianças, este não se associou ${ }^{18,23}$ ou associou-se negativamente ${ }^{20,21}$ ao desenvolvimento da linguagem.

É difícil dissociar se os efeitos dos DD sobre o desenvolvimento da linguagem se devem ao tempo ou ao conteúdo. Ainda que a maioria dos estudos apresentados apenas se foque num destes parâmetros, não podemos excluir a possibilidade do tempo de exposição se associar a piores desempenhos linguísticos como uma ex- 
pressão da exposição a conteúdos que prejudiquem o desenvolvimento das capacidades linguísticas. ${ }^{22}$

Televisão, smartphone, tablet: o mesmo efeito?

A maioria dos estudos que encontramos centra-se na análise dos efeitos da televisão. Dos 10 estudos analisados, três ${ }^{18,25,26}$ incorporaram DD móveis, contudo os resultados não foram separados por tipo de dispositivo. Assim, é impossível concluir qual é o efeito específico dos novos DD. Os estudos mostram que as crianças usam os smartphones e os tablets para visualizar vídeos de forma semelhante ao uso de DVDs e televisão. Contudo, os smartphones e os tablets são portáteis, podendo ser usados fora de casa, e são mais interativos, pelo que é expectável que os efeitos não sejam completamente sobreponíveis.

O que causa a associação negativa entre o consumo de dispositivos digitais e o desenvolvimento da linguagem?

O tempo de utilização de DD em idade pré-escolar parece depender de vários fatores. Entre outros, menores rendimentos familiares, ${ }^{2,12}$ menores níveis de educação dos pais ${ }^{2}$ e depressão materna ${ }^{32}$ associam-se consistentemente a tempos de exposição acrescidos. Crianças de estratos socioeconómicos mais desfavorecidos passam aproximadamente mais 90 minutos por dia em frente a DD do que crianças de estratos superiores. ${ }^{2}$ Paralelamente, filhos de pais com menor progressão académica têm aproximadamente mais 75 minutos de tempo de ecrã que filhos de pais com um nível de educação superior. ${ }^{2}$ Já a quantidade e qualidade de estimulação cognitiva no meio familiar (acesso a brinquedos educativos e tempo dedicado a ler com as crianças, por exemplo) associam-se negativamente à exposição a DD. ${ }^{32}$ Por este motivo, os estudos incluídos nesta revisão controlaram a sua análise para estas e outras variáveis de confundimento, como a estrutura familiar, ordem de nascimento e hábitos de leitura dos pais. A maioria dos estudos encontrou um efeito independente do uso de DD no desenvolvimento da linguagem, sugerindo um efeito causal. $\bigcirc$ mecanismo por detrás desta associação é desconhecido, e várias hipóteses têm sido apresentadas para a explicar. Uma hipótese é que outras variáveis de confundimento importantes não tenham sido controladas nestes estudos. Setenta porcento dos pais refere deixar as crianças brincar com dispositivos móveis para que consigam realizar as suas tarefas e 65\% para que as consigam acalmar em público. ${ }^{12}$ Este tipo de utilização pode ser mais frequente em pais mais impacientes, menos atentos ou simplesmente mais ocupados, que possam também não saber estimar com tanta precisão as palavras que os seus filhos conhecem. ${ }^{23}$ Assim, o estilo de parentalidade e outras possíveis variáveis podem justificar os resultados.

Considerando que existe uma associação entre a exposição e o resultado primário em estudo, esta pode advir do efeito direto da visualização de conteúdo via ecrã no desenvolvimento cognitivo. Contrariamente ao que seria de esperar, as imagens de programas para crianças em idade pré-escolar correm a um ritmo superior (com animações mais rápidas e com mais mudanças de cenários e de personagens), quando comparadas com programas desenhados para crianças mais velhas ou adultos, ${ }^{24,33} \mathrm{O}$ que pode constituir um excesso de estimulação visual e auditiva com potencial nocivo. ${ }^{4}$

Outra hipótese é que o efeito deletério do uso de DD no desenvolvimento da linguagem possa ser causado não pelo tempo dedicado aos DD em si, mas pela redução consequente do tempo em outras atividades. Muitos estudos apontam para a hipótese de que o tempo que poderia ser alocado a interações com adultos, a tempo recreativo com brinquedos, a ler ou a outras atividades cognitivamente enriquecedoras é redirecionado, sendo passado em frente a um ecrã. ${ }^{22,30,34}$ Ler para uma criança, por exemplo, apresenta uma associação inversa com o tempo dedicado a ver televisão ${ }^{35}$ e, apesar de não parecer ter um efeito marcado nas capacidades literárias da criança em idade pré-escolar, ${ }^{36}$ associa-se a melhores hábitos de leitura mais tarde, relacionados com melhor compreensão do conteúdo escrito e maior prazer pela leitura. ${ }^{37}$

Uma outra hipótese é que os DD afetem negativamente a qualidade da interação pais-crianças. Um estudo observacional japonês com uma pequena amostra $(n=14)$, com o objetivo de estudar o comportamento dos pais para com os filhos mediante uma televisão ligada ou desligada, mostrou um decréscimo significativo na quantidade de frases proferidas com a televisão ligada e no número de elementos de cada frase. ${ }^{38}$ De modo similar, também ocorre diminuição do número e duração das vocalizações das crianças e das conversas entre pais e filhos. ${ }^{39}$ Mesmo sem a atenção focada na televisão, o facto de estar ligada parece encurtar a duração dos episódios de brincadeira e da atenção focada durante os mesmos. 34

Finalmente, alguns trabalhos ${ }^{21,23}$ consideram a possibilidade de causalidade inversa, ou seja, em que é a perturbação da linguagem que causa o maior consumo de DD, tendo em conta que pais preocupados com a evolução inferior dos seus filhos podem recorrer a este tipo de conteúdos pelo potencial educativo que alegam. Contudo, na nossa revisão excluímos os estudos que incluíam 
crianças com perturbações da linguagem, o que invalida essa possibilidade.

Como pode a utilização de dispositivos digitais ser benéfica?

Nos estudos incluídos nesta revisão, encontramos alguns resultados que sugerem que o uso de DD pode beneficiar a aquisição de competências linguísticas.21,22,27 Assim, é importante perceber de que forma os devemos utilizar para obter melhores resultados, até porque o futuro das crianças de hoje requer proficiência digital. Vários estudos enfatizaram a relevância da interação ao vivo no processo de aquisição da linguagem através de DD. ${ }^{40,41}$ Em 2009, Roseberry et al mostraram que crianças de apenas 30-35 meses conseguem aprender verbos através de vídeos educativos, desde que acompanhados por interação social presencial que os reforce. ${ }^{42}$ Mendelsohn et al estudaram o papel das interações verbais dos pais durante a exposição dos seus filhos a DD aos 6 meses e o desenvolvimento da linguagem destas crianças aos 14 meses. Os resultados mostram associações negativas apenas na ausência de interações verbais aos 6 meses. ${ }^{43}$ Assim, parece ser recomendável a utilização conjunta e acompanhada pelos pais.

\section{CONCLUSÃO}

Mediante os estudos analisados, concluímos que o uso de DD parece prejudicar ligeiramente o desenvolvimento da linguagem em crianças em idade pré-escolar. Esta associação está presente não apenas para conteúdos não dirigidos para crianças como também para programas educativos infantis. Assim, os benefícios decorrentes da exposição de crianças em idade pré-escolar aos media não parecem superar os potenciais riscos.

Apesar do número limitado de estudos nesta área, a evidência atual mostra-nos que importa acautelar pais, cuidadores, professores e profissionais de saúde quanto à importância da inclusão sensata e parcimoniosa destes dispositivos no dia-a-dia das suas crianças. A realização de mais estudos poderia reforçar estas conclusões e permitiria esclarecer as dúvidas que ainda permanecem. Considerando a constante evolução dos DD, com impacto evidente nos padrões de consumo, esta necessidade assume particular relevância.

CONFLITOS DE INTERESSE: Os autores declaram não ter qualquer conflito de interesse na realização do presente trabalho.

FONTES DE FINANCIAMENTO: Não houve qualquer fonte de financiamento na realização do presente trabaIho.
PROVENIÊNCIA E REVISÃO POR PARES: Não comissionado; revisão externa por pares.

CONFLICTS OF INTEREST: The authors declare that they have no conflicts of interest.

FINANCIAL SUPPORT: This work has not received any contribution, grant or scholarship.

PROVENANCE AND PEER REVIEW: Not commissioned; externally peer reviewed.

\section{REFERÊNCIAS}

1. Evolution of the Mobile Phone [página da Internet]; 20082018 [acedido em Fev 2019]. Disponível em: https://www. tigermobiles.com/evolution/\#zeroPhone.

2. Rideout V. The Common Sense Census: Media Use By Age Zero to Eight. San Francisco: Common Sense Media; 2017.

3. Deng T, Kanthawala S, Meng J, Peng W, Kononova A, Hao Q, et al. Measuring smartphone usage and task switching with log tracking and self-reports. Mobile Media Commun. 2018;7:323. doi: $10.1177 / 2050157918761491$.

4. Christakis DA, Ramirez JSB, Ferguson SM, Ravinder S, Ramirez JM. How early media exposure may affect cognitive function: A review of results from observations in humans and experiments in mice. Proc Natl Acad Sci U S A. 2018;115:98518. doi: 10.1073/pnas.1711548115.

5. Radesky JS, Christakis DA. Increased screen time: implications for early childhood development and behavior. Pediatr Clin North Am. 2016;63:827-39. doi: 10.1016/j.pcl.2016.06.006.

6. Cain N, Gradisar M. Electronic media use and sleep in school-aged children and adolescents: A review. Sleep Med. 2010;11:735-42. doi: 10.1016/j.sleep.2010.02.006.

7. Lissak G. Adverse physiological and psychological effects of screen time on children and adolescents: Literature review and case study. Environ Res. 2018;164:149-57. doi: 10.1016/j.envres.2018.01.015.

8. Rocha G. Electronic screens and children's vision: development of a joint position statement. Can J Ophthalmol. 2018;53:307. doi: 10.1016/j.jcjo.2018.06.014.

9. Ruest S, Gjelsvik A, Rubinstein M, Amanullah S. The inverse relationship between digital media exposure and childhood flourishing. J Pediatr. 2018;197:268-74 e2. doi: 10.1016/j. jpeds.2017.12.016.

10. Twenge JM, Campbell WK. Associations between screen time and lower psychological well-being among children and adolescents: Evidence from a population-based study. Prev Med Rep. 2018;12:271-83. doi: 10.1016/j.pmedr.2018.10.003.

11. Robinson TN, Banda JA, Hale L, Lu AS, Fleming-Milici F, Calvert SL, et al. Screen media exposure and obesity in children and adolescents. Pediatrics. 2017;140(Suppl 2):S97-S101. doi: 10.1542/peds.2016-1758K.

12. Kabali HK, Irigoyen MM, Nunez-Davis R, Budacki JG, Mohanty SH, Leister KP, et al. Exposure and use of mobile media devices by young children. Pediatrics. 2015;136:1044-50. doi: 10.1542/peds.2015-2151.

13. Black MM, Walker SP, Fernald LCH, Andersen CT, DiGirolamo AM, Lu CL, et al. Early childhood development coming of age: science through the life course. Lancet. 2017;389:77-90. doi: 10.1016/S0140-6736(16)31389-7.

14. Fox SE, Levitt P, Nelson CA. How the timing and quality of early experiences influence the development of brain ar- 
chitecture. Child Dev. 2010;81:28-40. doi: DOI 10.1111/j. 1467-8624.2009.01380.x.

15. Kolb B, Mychasiuk R, Gibb R. Brain development, experience, and behavior. Pediatr Blood Cancer. 2014;61:1720-3. doi: 10.1002/pbc.24908.

16. Feldman HM. Using the language characteristics of clinical populations to understand normal language development. Pediatr Clin North Am. 2007;54:585-607, viii. doi: 10.1016/j. pcl.2007.02.006.

17. AAP Council of Communications and Media. Media and Young Minds. Pediatrics. 2016;138. doi: 10.1542/peds.2016-2591.

18. Duch H, Fisher EM, Ensari I, Font M, Harrington A, Taromino $C$, et al. Association of screen time use and language development in Hispanic toddlers: a cross-sectional and longitudinal study. Clin Pediatr. 2013;52:857-65. doi: 10.1177/0009922813492881.

19. Schmidt ME, Rich M, Rifas-Shiman SL, Oken E, Taveras EM. Television viewing in infancy and child cognition at 3 years of age in a US cohort. Pediatrics. 2009;123:e370-5. doi: 10.1542/ peds.2008-3221.

20. Tomopoulos S, Dreyer BP, Berkule S, Fierman AH, Brockmeyer C, Mendelsohn AL. Infant media exposure and toddler development. Arch Pediatr Adolesc Med. 2010;164:1105-11. doi: 10.1001/archpediatrics.2010.235.

21. Wright JC, Huston AC, Murphy KC, St Peters M, Pinon M, Scantlin $\mathrm{R}$, et al. The relations of early television viewing to school readiness and vocabulary of children from low-income families: the early window project. Child Dev. 2001;72:134766.

22. Zimmerman FJ, Christakis DA. Children's television viewing and cognitive outcomes: a longitudinal analysis of national data. Arch Pediatr Adolesc Med. 2005;159:619-25. doi: 10.1001/archpedi.159.7.619.

23. Zimmerman FJ, Christakis DA, Meltzoff AN. Associations between media viewing and language development in children under age 2 years. J Pediatr. 2007;151:364-8. doi: 10.1016/j. jpeds.2007.04.071.

24. Okuma K, Tanimura M. A preliminary study on the relationship between characteristics of TV content and delayed speech development in young children. Infant Behav Dev. 2009;32:31221. doi: 10.1016/j.infbeh.2009.04.002.

25. Moon JH, Cho SY, Lim SM, Roh JH, Koh MS, Kim YJ, et al. Smart device usage in early childhood is differentially associated with fine motor and language development. Acta Paediatr. 2019;108:903-10. doi: 10.1111/apa.14623.

26. Lin LY, Cherng RJ, Chen YJ, Chen YJ, Yang HM. Effects of television exposure on developmental skills among young children. Infant Behav Dev. 2015;38:20-6. doi: 10.1016/j.infbeh.2014.12.005.

27. Castles A, McLean GM, Bavin E, Bretherton L, Carlin J, Prior $M$, et al. Computer use and letter knowledge in pre-school children: a population-based study. J Paediatr Child Health. 2013;49:193-8. doi: 10.1111/jpc.12126.

28. Chonchaiya W, Pruksananonda C. Television viewing associates with delayed language development. Acta Paediatr. 2008;97:977-82. doi: 10.1111/j.1651-2227.2008.00831.x.

29. Garrison M, Christakis D. A Teacher in the Living Room? Educational Media for Babies, Toddlers, and Preschoolers. Menlo Park: The Henry J. Kaiser Family Foundation; 2005.

30. Wartella E, Rideout VJ, Vandewater EA. Zero to Six: Electronic Media in the Lives of Infants, Toddlers and Preschoolers. Menlo Park: Kaiser Family Foundation; 2003.
31. Linebarger DL, Walker D. Infants' and toddlers' television viewing and language outcomes. Am Behav Sci. 2016;48:624-45. doi: 10.1177/0002764204271505.

32. Duch H, Fisher EM, Ensari I, Harrington A. Screen time use in children under 3 years old: a systematic review of correlates. Int J Behav Nutr Phys Act. 2013;10:102. doi: 10.1186/14795868-10-102.

33. Goodrich SA, Pempek TA, Calvert SL. Formal production features of infant and toddler DVDs. Arch Pediatr Adolesc Med. 2009;163:1151-6. doi: 10.1001/archpediatrics.2009.201.

34. Schmidt ME, Pempek TA, Kirkorian HL, Lund AF, Anderson DR. The effects of background television on the toy play behavior of very young children. Child Dev. 2008;79:1137-51. doi: 10.1111/j.1467-8624.2008.01180.x.

35. Khan KS, Purtell KM, Logan J, Ansari A, Justice LM. Association Between Television Viewing and Parent-Child Reading in the Early Home Environment. J Dev Behav Pediatr. 2017;38:5217. doi: 10.1097/dbp.0000000000000465.

36. Napoli AR, Purpura DJ. The home literacy and numeracy environment in preschool: Cross-domain relations of parent-child practices and child outcomes. J Exp Child Psychol. 2018;166:581-603. doi: 10.1016/j.jecp.2017.10.002.

37. Senechal M. Testing the Home Literacy Model: Parent Involvement in kindergarten is differentially related to grade 4 reading comprehension, fluency, spelling, and reading for pleasure. Sci Stud Reading. 2006;10:59-87. doi: 10.1207/ s1532799xssr1001_4.

38. Tanimura M, Okuma K, Kyoshima K. Television viewing, reduced parental utterance, and delayed speech development in infants and young children. Arch Pediatr Adolesc Med. 2007;161:618-9. doi: 10.1001/archpedi.161.6.618-b.

39. Christakis DA, Gilkerson J, Richards JA, Zimmerman FJ, Garrison MM, Xu D, et al. Audible television and decreased adult words, infant vocalizations, and conversational turns: a population-based study. Arch Pediatr Adolesc Med. 2009;163:5548. doi: 10.1001/archpediatrics.2009.61.

40. Krcmar M, Grela B, Lin K. Can Toddlers Learn Vocabulary from Television? An Experimental Approach. Media Psychol. 2007;10:41-63.

41. Naigles LR, Bavin EL, Smith MA. Toddlers recognize verbs in novel situations and sentences. Dev Sci. 2005;8:424-31. doi: 10.1111/j.1467- 7687.2005.00431.x.

42. Roseberry S, Hirsh-Pasek K, Parish-Morris J, Golinkoff RM. Live action: can young children learn verbs from video? Child Dev. 2009;80:1360-75. doi: 10.1111/j.1467-8624.2009.01338.x.

43. Mendelsohn AL, Brockmeyer CA, Dreyer BP, Fierman AH, Berkule-Silberman SB, Tomopoulos S. Do Verbal interactions with infants during electronic media exposure mitigate adverse impacts on their language development as toddlers? Infant Child Dev. 2010;19:577-93. doi: 10.1002/icd.711. 